\title{
Impacto de la infraestructura en las operaciones logísticas Gestión de carga y entrega de mercancías
}

\author{
Impact of infrastructure on logistic operations \\ Loading and delivery management
}

Gabriela Maraví $^{1}$, Diego Matuk $^{2}$ y Mario Chong
Recibido:

Resumen. - El rápido crecimiento de la población urbana ha generado el incremento de la demanda de bienes y servicios; asimismo, de las operaciones de distribución para satisfacer las necesidades propias de los clientes actuales. Ante esta situación, hay aspectos que representan un reto para la distribución de mercancías en las ciudades: la infraestructura en logística con su gestión de operaciones de carga y descarga, las entregas de mercancías, las regulaciones adecuadas, las vías, la señalización y los espacios públicos para el desenvolvimiento de las actividades humanas. Las bahías de carga y descarga son espacios de estacionamiento públicos o privados. Están destinados para realizar operaciones de abastecimiento y logística inversa de la cadena de suministros en los centros y puntos de distribución por vehículos de diversas características, de acuerdo con la operación de diferentes productos y servicios. Estas zonas facilitan la entrega de mercancías ya que reducen el impacto en la congestión del tráfico vehicular y favorecen la sostenibilidad ambiental de la ciudad.

El presente artículo da a conocer las características de la infraestructura logística de la zona comercial de Chosica, ubicada en el distrito de Lurigancho en el departamento de Lima (Perú). También presenta la problemática de la ciudad, asociada a la ausencia de una política de carga y descarga de mercancías; del mismo modo, propone una integración de iniciativas para mejorar la distribución de mercancías en la zona. Para la caracterización de Chosica se utilizó la metodología del kilómetro cuadrado $\left(\mathrm{km}^{2}\right)$, propuesta por el Massachusetts Institute of Technology (MIT) con el inventario de comercios (generadores de carga), regulaciones y vías; y la observación y análisis de sus dinámicas y comportamiento en el frente más denso con sus disrupciones, entregas de mercancías y vehículos. De acuerdo con la caracterización de las condiciones de esta zona comercial y su generación de carga, se proponen políticas públicas y mejores prácticas para el desarrollo urbano y sostenibilidad de la ciudad. Los resultados muestran deficiencia en la infraestructura y en las instalaciones físicas para la operación de los flujos de la cadena de suministros; por otro lado, su influencia en el transporte, el inventario y el aprovisionamiento, desde la perspectiva de los operadores logísticos, se presentan con una pérdida en la eficiencia en el reparto de mercancías, reflejada en el precio final al consumidor. Las propuestas para la gestión de cargas y entregas de

\footnotetext{
${ }^{1}$ Universidad del Pacífico, e-mail ga.maraviv@up.edu.pe ORCID 0000-0003-0732-7658

${ }^{2}$ Universidad del Pacífico e-mail dm.matukb@up.edu.pe ORCID 0000-0002-0749-2920

${ }^{3}$ Universidad del Pacífico e-mail m.chong@up.edu.pe ORCID 0000-0002-1231-0992
} 
mercancías presentan el uso multimodal del espacio urbano y la adaptación de cada frente con el objetivo de mantener un equilibrio entre las necesidades de abastecimiento de los comercios y la demanda de los ciudadanos, la movilidad de la ciudad y la sostenibilidad de este ambiente creado por el ser humano.

Palabras clave: operaciones logísticas; distribución de mercancías; bahías de carga y descarga.

Abstract. -The rapid growth of the urban population generates an increase the demand for goods and services, and the distribution operations to meet their requirements. In these cases, the infrastructure in logistics operations with the adequate loading and unloading operations, the delivery management, the regulations, the ways, the regulations and the public spaces layout for human activities, represents a challenge for the goods distribution in the cities. The loading and unloading docks are public or private parking spaces for the efficient supply and inverse logistics supply operations, in the distribution centers and points of distribution by vehicles of different classes, according the operation characteristics of the products and services. These zones facilitate the delivery of goods, reduce the impact on traffic congestion and the environmental sustainability of the city.

This paper characterizes the logistics infrastructure in the commercial zone of Chosica, located in the district of Lurigancho-Chosica, department of Lima, Peru. This paper presents the city characteristics with the absence of a freight loading and unloading policies and proposes the integration of initiatives to improve the goods distribution in the area. The methodology used for the characterization of Chosica was the methodology of square kilometer $\left(\mathrm{km}^{2}\right)$ proposed by the Massachusetts Institute of Technology (MIT) with the commercial inventory (load generators), regulations and roads; and the observation and analysis of its dynamics and behavior on the densest front with its disruptions, deliveries and vehicles. According to the conditions characterization of this commercial zone and its cargo generation, public policies and best practices for urban development and city sustainability are proposed. The results show a deficiency in the infrastructure and the physical facilities for an efficient operation of supply chain flows, their influences on transportation, inventory and supply from the perspective of logistics operators with an inefficient distribution of goods, reflected in the price. The proposals for loads and deliveries of goods management present the multimodal use of the urban space and the review of each front in order to maintain a balance between the supply of the commerce and the demand of the citizens, the mobility of the city and the sustainability of this environment created by the human being.

Keywords: logistic operations; distribution of goods; loading and unloading docks. 
1. Introducción. - El crecimiento de la población en las ciudades genera un incremento de la demanda por bienes y servicios. En virtud de ello, el proceso de carga y descarga de mercancías es una función importante y frecuente en el sistema de transporte urbano. El aumento de las necesidades se evidencia mediante la cantidad de personas en las zonas urbanas: en el año 2000, ascendía a 46,70\%; en 2007, 50,15\% y para el 2017, 54,74\% o más de 4000 millones de personas [1]. Debido al aumento considerable de pobladores en zonas urbanas, y su demanda proporcional de productos y servicios, las operaciones logísticas se convierten en un factor importante y complejo para atender estas solicitudes. Las ciudades soportan una mayor circulación de transporte de carga, la cual representa entre el $10 \%$ y el $20 \%$ del tráfico interno actualmente [2]. La interacción entre el suministro y la demanda de productos ha evolucionado de manera exponencial al ritmo de los negocios, con una personalización creciente en las entregas y en la digitalización de las operaciones. A pesar de los cambios presentados, estos ambientes de negocios están sujetos a las condiciones de las ciudades: la infraestructura (vías, estacionamientos, áreas de carga y descarga, almacenes), la señalización y el espacio público con una dinámica diferente; lo cual, representa un problema para la distribución de mercancías [3], considerando sus externalidades como la congestión de tráfico, ruido, contaminación ambiental, inseguridad vial y disminución de la competitividad de las operaciones logísticas, además de sus actividades relacionadas [4].

La función importante del transporte de carga en la actividad humana, la economía urbana y sus impactos se expresan en la necesidad de ser considerado en los planes de movilidad [5]; a pesar de ello, ha sido descuidado por los modelos, en las estrategias de transporte y en la planificación regional [6]. No obstante, los operadores de carga cumplen con proporcionar los bienes solicitados en el lugar y momento requerido, con soluciones a la medida sin una visión sistemática de la congestión; y tienden a implementarse sin mayor coordinación y regulación [7]. Las políticas públicas locales, relacionadas al transporte de mercancías, son escasas [4]. Generalmente, no es considerado como flujo en la movilidad urbana, el cuál debería organizarse de una manera más eficiente como uno de los actores en el espacio público: peatones, transporte público, transporte privado (en diferentes medios), comercios y otros [8].

Las zonas de carga y descarga de mercancías, llamadas bahías de carga y descarga, permiten la entrega de mercancías de manera rápida, sin causar interrupciones, respetando las regulaciones de transporte [9]. La ausencia de dichas normas permite que las operaciones se realicen en las vías de transporte, causando congestión vehicular [10]. En las ciudades, la escasez de bahías de carga y descarga constituyen una característica común: menos del $10 \%$ de los segmentos de la calle cuentan con estos espacios. Esto indica la poca atención a una infraestructura de carga urbana adecuada [3]. Por otro lado, en algunos lugares en el mundo sucede lo siguiente: la ciudad de París cuenta con 10 000 áreas de carga y descarga; Barcelona, con 8 000; Buenos Aires posee 750 áreas y Belo Horizonte 550 en la región central de la ciudad [11]. A pesar de estos números, la demanda creciente de los ciudadanos y el aumento desproporcional de los espacios públicos conlleva a una demanda de estacionamientos especiales, superior a la capacidad linear de las calles [12]; en consecuencia, los transportistas se ven obligados a estacionarse en doble fila y a pagar multas por tal acción. Un claro ejemplo de esto último es Nueva York, cada unidad de transporte tiene entre $\$ 500$ y $\$ 1000$ por mes en multas respecto al estacionamiento en zonas indebidas [13]. Si en ciudades con un

Memoria Investigaciones en Ingeniería, 2019. $\mathrm{N}^{\circ} 17(1) . p p$ 31-46

https://doi.org/10.36561/ING.17.3

ISSN 2301-1092 • ISSN (en línea) 2301-1106 
desarrollo urbano amplio y planeamiento sofisticado la situación sigue siendo precaria, no puede esperarse que la situación en países en desarrollo sea mejor.

Perú es un país que, durante la última década, se encuentra en un ritmo de crecimiento económico constante. La tasa de población urbana alcanza alrededor del $80 \%$ y la densidad urbana llega a un promedio de 3500 personas por $\mathrm{km}^{2}$. Lima, su capital, alberga el 32\% de la población del país con más de 10 millones de habitantes, con una demanda creciente de servicios públicos eficientes, atractivos para la inversión, además y que posibiliten una dinámica y sostenida actividad económica adecuada [1]. Una parte de estos servicios públicos se refieren a los servicios de agua, luz, comunicaciones y transporte.

Sin embargo, cabe señalar que la forma de operar, en términos logísticos, sobre todo en áreas comerciales es desorganizada. Esto se refleja en la falta de políticas públicas integradas, con el acuerdo de las partes implicadas y afectadas [14-15]. Las operaciones logísticas de distribución de mercancías en la ciudad de Lima se dan de forma desordenada, crece la congestión vehicular y se producen los embotellamientos que implican una pérdida de competitividad del país. Según el índice de competitividad 2018 del Foro Económico Mundial, Lima estaba en el puesto 63, a comparación del puesto 60 del año 2017. Según el índice de la empresa Tomtom, es la tercera ciudad más congestionada del mundo, detrás de Mumbai y Bogotá, con un índice de congestión del $58 \%$ [16]. La ciudad de Lima cuenta con pocos espacios destinados a la carga y descarga de mercancías; por ejemplo, el distrito de Miraflores tiene un área de 9,62 $\mathrm{km}^{2}$ [17] y cuenta con 148 bahías de carga y descarga privadas [11]. La determinación de soluciones innovadoras de logística es importante para mejorar la distribución de mercancías en las áreas urbanas altamente congestionadas. En el presente artículo, se caracteriza la infraestructura logística de la zona comercial de Chosica en la ciudad de Lima (Perú), se analiza la problemática asociada a la falta de bahías de carga y descarga, así como se propone un conjunto de iniciativas para mejorar la distribución de mercancías en la zona.

2. Marco teórico. - La logística urbana es un eslabón de la cadena del transporte de mercancías, la cual tiene como objetivo comprender, estudiar y analizar las diferentes organizaciones con el propósito de optimizar las actividades de transporte [18], cuya complejidad se evidencia conforme crece la población y sus requerimientos [19]. Según Dablanc [8] y Hall y Hesse [2], este traslado de mercancías hacía el consumidor es fundamental para la cadena de suministro, pero no está considerado en el proceso de planeamiento urbano; en consecuencia, ha obligado a las empresas proveedoras a implementar medidas particulares, no siempre alineadas con el contexto urbano para poder realizar la distribución de mercancías [3]. A pesar de esto, las políticas públicas tienen como objetivo, maximizar los beneficios sociales netos con relación a las actividades de carga; a la vez que minimizan las externalidades negativas del tráfico y maximizan los flujos de carga confiables [20]. Sin embargo, la inclusión de medidas eficientes es limitada, no solo por la fragmentación de los actores del sistema urbano [4] y la importancia, sino también por el sesgo existente al transporte de pasajeros [21-22].

Holguín-Veras et. al [20] propone el proceso de toma de decisiones para el transporte urbano de mercancías con una metodología para encontrar soluciones y plantear acciones que permitan mejorar el transporte de carga. Este proceso puede ser utilizado en cualquier área geográfica, en varios tipos de planificaciones, en ventanas de tiempo de diversas duraciones y se estructura en los siguientes pasos: delimitación del

Memoria Investigaciones en Ingeniería, 2019. $\mathrm{N}^{\circ} 17(1) . p p$ 31-46

https://doi.org/10.36561/ING.17.3

ISSN 2301-1092 • ISSN (en línea) 2301-1106 
problema y sus causas raíz; definición del objetivo y sus medidas de desempeño; identificación de las potenciales soluciones al problema; selección de las opciones más viables; creación de un plan de acción para su implementación y una posible prueba piloto. Los autores presentan una serie de políticas públicas de transporte como la mejora e innovación de la infraestructura, el desarrollo de clústeres de carga, rampas para carretillas, restricciones de carga y estacionamiento, entre otras. Por su parte, Blanco y Merchán [4] presentan un conjunto de políticas públicas y soluciones de logística urbana observadas en zonas de alta congestión de Latinoamérica: restricciones al acceso de los vehículos de transporte de mercancías según sus dimensiones y el tamaño de su carga, ventanas de tiempo para las operaciones logísticas, bahías de carga y descarga de mercancías, así como espacios de transbordo de carga.

Con relación al transporte de carga, Blanco y Merchán [4] consideran que la implementación de zonas de estacionamiento destinadas para los vehículos de carga son las políticas de infraestructura de menor costo y de sencilla ejecución. Para ellos, estacionar a distancia de los locales conlleva a tiempos más largos de entrega y al aumento de sanciones contractuales. Aumentar la capacidad de estacionamiento, además de áreas para carga y descarga permite reducir la congestión a un costo bajo, como confirma Nourinejad et al. [23] en un estudio de simulación de tráfico que evaluó los impactos de estrategias alternativas de estacionamiento de carga. Adicionalmente, Holguín Veras y Sánchez-Diaz [24] prueban la efectividad de una política de entregas fuera de horario (de las 15:00 P.M. a 06:00 A.M.), la cual puede reducir hasta en un $20 \%$ el tráfico de carga. Además, este tipo de acciones permite organizar las entregas, reducir la cantidad de emisiones y costos de transporte [25].

La ciudad de Tokio es uno de los ejemplos de implementación de estos espacios. Con una ordenanza de estacionamiento fuera de la vía pública en el año 2002 obligó a todos los grandes almacenes, oficinas y bodegas a brindar bahías de carga y descarga cuando excedían una superficie mayor a $2.000 \mathrm{~m} 2$. La ciudad de París impone la existencia de una bahía cada 100 metros en las calles de la ciudad [8]. Por su parte, Nueva York aumentó la asignación de estacionamiento para vehículos comerciales [20]. Otra iniciativa fue la implementación de un sistema de reservas para estacionamientos de carga y descarga en la ciudad de Toyota (Japón); así se redujo la cantidad de vehículos de transporte de carga en el tráfico en un 56\% aproximadamente [26].

Sin embargo, la literatura recién está discutiendo el planeamiento urbano basado en datos y no hay un consenso en la actualidad. Existe una carencia de teoría y metodologías apropiadas para el manejo apropiado de transporte urbano de mercancías [27-28], los cuales son necesarios para una correcta implementación de políticas [29]. Estos modelos en su mayoría, como lo explica Mancini [30], tienden a ser optimizaciones basadas en la carga de transporte. Pero estas solo son una parte de lo requerido. No hay una política actual que permita satisfacer las demandas y requerimientos del transporte de carga, con relación a los diferentes efectos que tienen cada una [30]. Por lo tanto, es necesario integrar no solo la logística urbana, sino que, en adición, las políticas dirigidas a estos cubran el ámbito precisado.

3. Metodología. - Para la caracterización urbana de la zona comercial del distrito de Lurigancho-Chosica, se utilizó la metodología del $\mathrm{km}^{2}$ propuesta por Blanco y explicada por Merchán [5] del Massachusetts Institute of Technology (MIT) [27], la cual propone la definición y posterior segmentación de la zona de estudio para luego

Memoria Investigaciones en Ingeniería, 2019. №17(1). pp 31-46

https://doi.org/10.36561/ING.17.3

ISSN 2301-1092 • ISSN (en línea) 2301-1106 
proceder a recolectar el inventario de la zona y la observación de un frente seleccionado. La Figura I presenta los pasos de la metodología.

La primera fase de la metodología propone un inventario de la zona con los siguientes formularios: inventario de comercios, regulaciones y vías. La segunda fase tiene el propósito de observar el desenvolvimiento del frente más denso, sus factores variables (no son fijos en el corto plazo) en un periodo para establecer la cantidad de disrupciones, número de entregas y el conteo del tráfico. La observación se realizó en una semana desde las 08:00 hasta las 17:00 horas, y los formularios usuarios fueron los siguientes: entregas, disrupciones y tráfico.

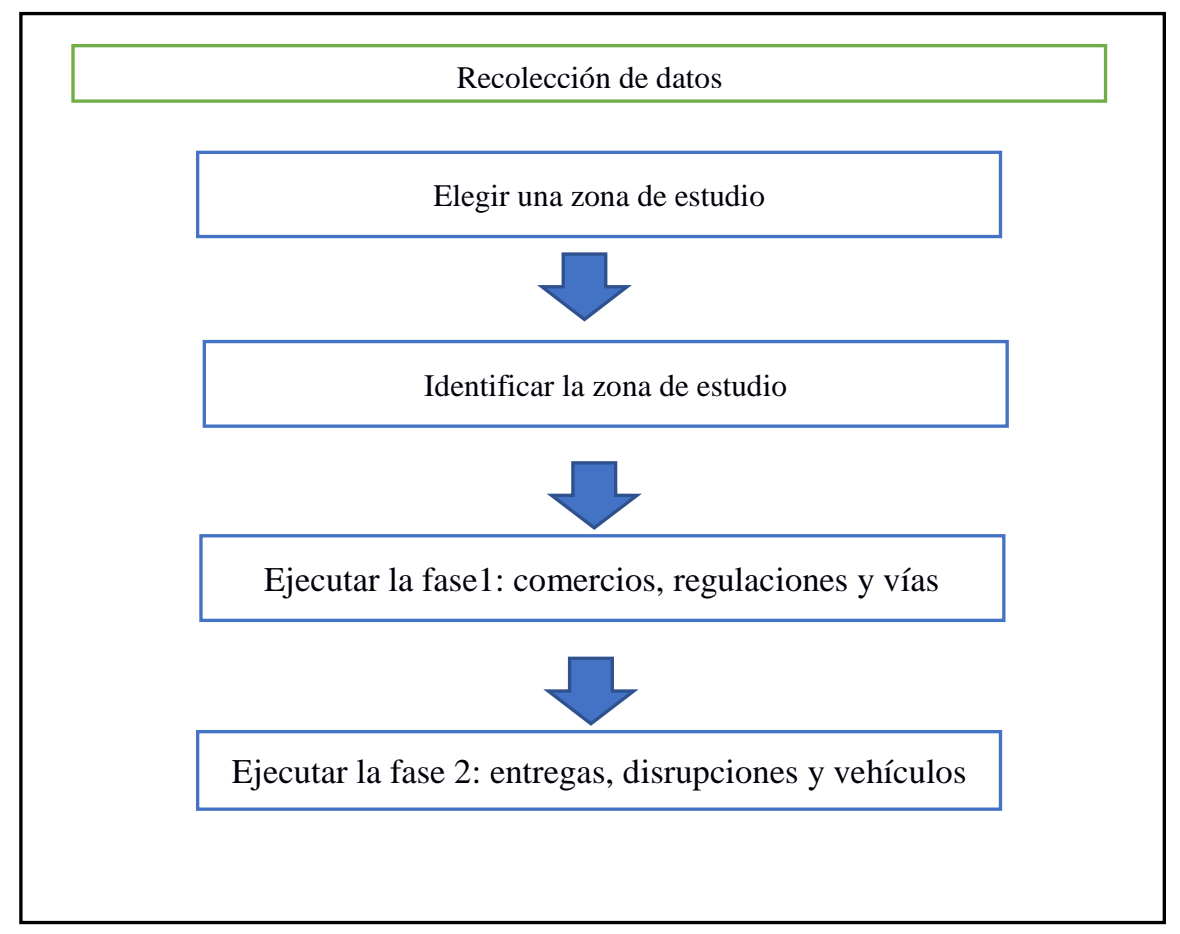

Figura I: Recolección de datos. Fuente: Massachusetts Institute of Technology [31]

4. Definición de la zona de estudio. -El área de aplicación de la metodología está ubicada en la zona comercial del distrito de Lurigancho-Chosica, en la ciudad de Lima (Perú) con una superficie de 236,47 km² [32]. La ciudad de Chosica, capital del distrito de Lurigancho-Chosica, se encuentra en la Carretera Central, vía de penetración a la sierra del Perú, con una población urbana de 173345 habitantes para el 2017 y una densidad de 733,05 habitantes/ $\mathrm{km}^{2}$ [33].

La metodología se aplicó en la zona comercial de Chosica, (Figura II) y en cuatro frentes (Figura III) [34]: dos frentes en el Jr. Libertad y dos frentes en la Av. Prolongación 28 de Julio. Los frentes se codificaron de la siguiente manera: 1, ubicado en la cuadra 1 del Jr. Libertad; 2, ubicado en la cuadra 2 del Jr. Libertad; 3, ubicado en la cuadra 7 de la Av. Prolongación 28 de Julio y 4, ubicado en la cuadra 7 de la Av. Prolongación 28 de Julio. 


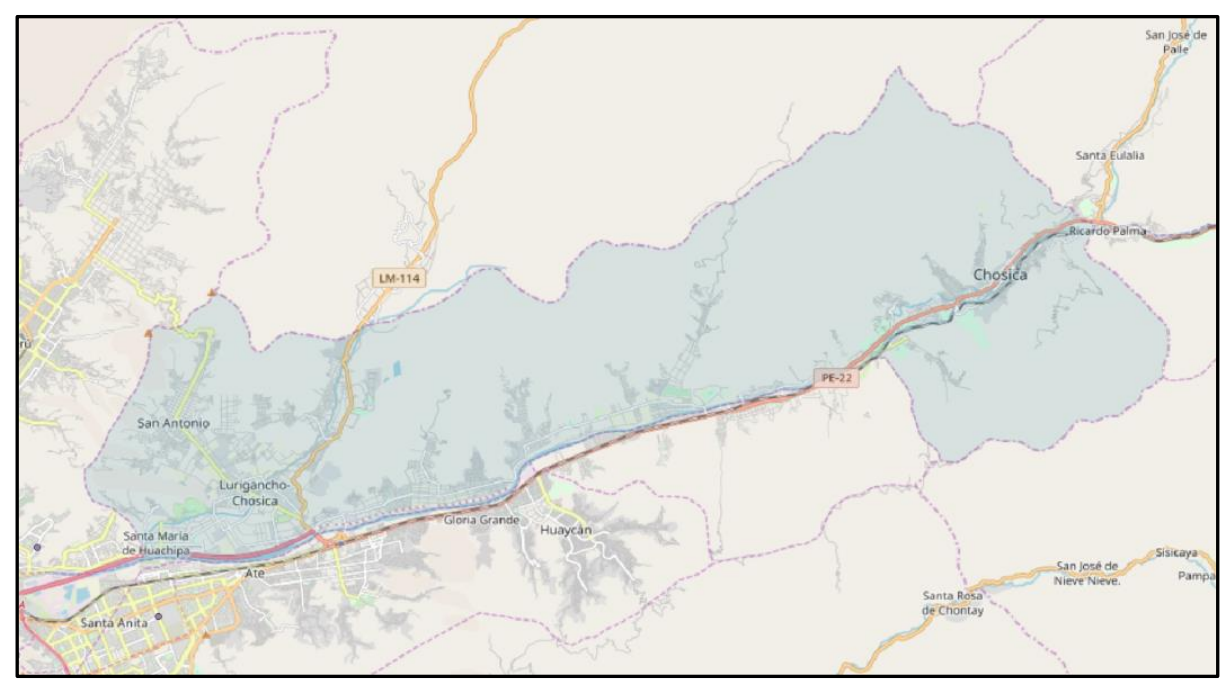

Figura II: Mapa del distrito de Lurigancho. Fuente: Open Street Maps[34]

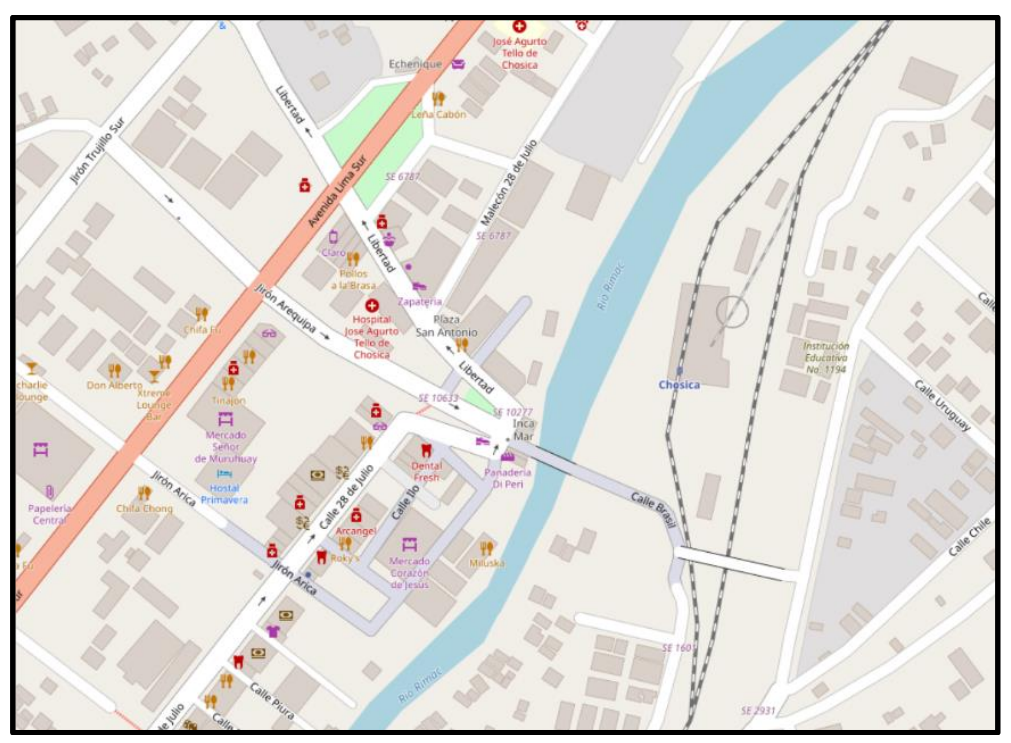

Figura III: Zona de estudio. Fuente: Open Street Maps [34]

\section{Resultados}

5.1. Inventario de comercios. - La zona comercial de Chosica tiene 53 comercios con una amplia variedad de rubros como se muestra en la Figura IV. El 22,64\% se clasificó como otros servicios, considerándose pequeños locales minoristas con un área aproximada de $40 \mathrm{~m}^{2}$, dedicados a las ventas de servicios como telefonía y asesorías profesionales. El 18,87\% se clasificó como otros retails o negocios minoristas dedicados al comercio de telas, libros, juguetes y productos diversos. También el 18,87\% de los comercios son negocios minoristas dedicados a la venta de productos de primera necesidad y alimentos. El 13,21\% está representado por farmacias independientes y dos cadenas de farmacias. El 11,32\% de los locales está compuesto por establecimiento de venta de comida clasificados en dos rubros: comida rápida y venta de menú. El 11,32\% 
se dedica a la venta de ropa y un 3,77\% representa pequeños supermercados con un área menor a $100 \mathrm{~m}^{2}$. Todos los comercios del área comercial carecen de una zona de carga y descarga, además de estacionamientos de los locales comerciales.

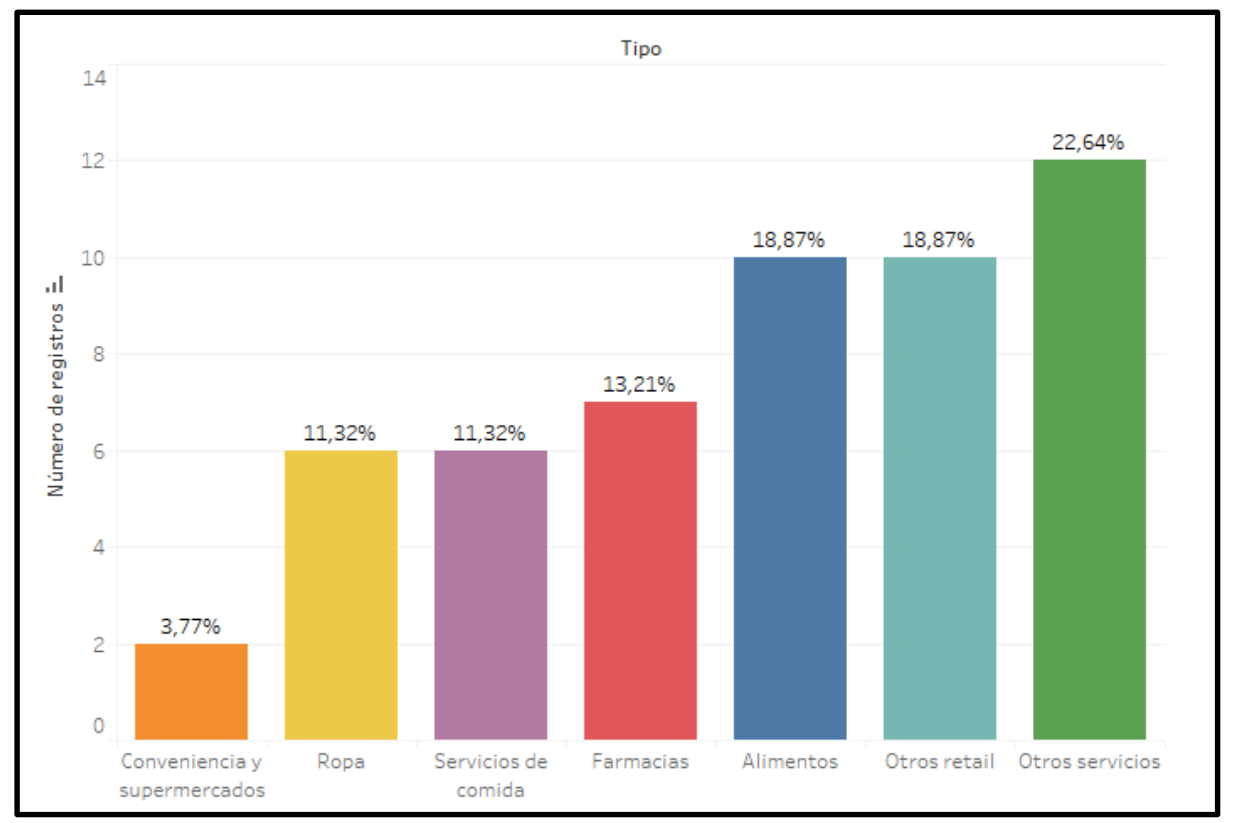

Figura IV: Proporción de tiendas según tipo de comercio. Fuente: Elaboración propia

5.2. Inventario de vías. - Las vías de las cuatro cuadras de la observación son de un sentido con dos carriles de circulación de vehículos. Se contabilizaron un total de treinta y cinco estacionamientos. Debido a la ausencia de las regulaciones y las bahías de carga y descarga, hay una competencia por el uso de los estacionamientos, así como del uso de uno de los carriles para estas operaciones, las mismas que ocasionan frecuentes congestiones vehiculares.

5.3. Inventario de regulaciones. - La ausencia de regulaciones fue una característica de atención en la primera fase. La única regulación fue la prohibición de estacionamiento al lado derecho de la vía, por el borde de la calzada o de las aceras pintadas de amarillo.

5.4. Seguimiento de entregas. - En las Figuras V-IX se analizan los frentes.

5.4.1. El primer frente fue el más concurrido, con un $50 \%$ de las entregas y una distancia promedio de 24,60 metros entre el vehículo y el establecimiento, la cual fue calculada comparando los metros de la ubicación del vehículo estacionado con los puestos, durante su abastecimiento. Los vehículos utilizados por los transportistas fueron camiones de cinco y ocho toneladas. En el primer frente, el 65\% de las entregas se realizaban entre las 8:00 y las 12:00 horas con una duración promedio de 39,21 minutos. El comercio con mayor número de entregas fue un establecimiento de venta de comidas: pollos a la brasa y papas procesadas.

5.4.2. El segundo frente tuvo un comportamiento similar en la mañana, con un horario adicional entre las 13:00 y las 16:00 horas con una duración promedio en la entrega de 31,25 minutos. El movimiento de carga es considerablemente menor. Entre los 
comercios, se encontraron tiendas, verdulerías y una pastelería. La distancia promedio de los vehículos a los establecimientos fue de tres metros.

5.4.3. El tercer frente tuvo entregas desde las 09:00 hasta las 16:10 horas con un promedio de 40,20 minutos por entrega. Un restaurante recibió el mayor número de entregas y una cadena de farmacias el menor número de entregas. La distancia promedio de carga fue de 10,20 metros.

5.4.4. El cuarto frente tuvo el $80 \%$ de las entregas entre las 09:00 y las 12:00 horas con una duración promedio de 42,55 minutos. Los negocios con mayor número de entregas fueron dos restaurantes con el suministro de pollos y papas procesadas. La distancia promedio de los vehículos a los comercios fue de 10,80 metros.

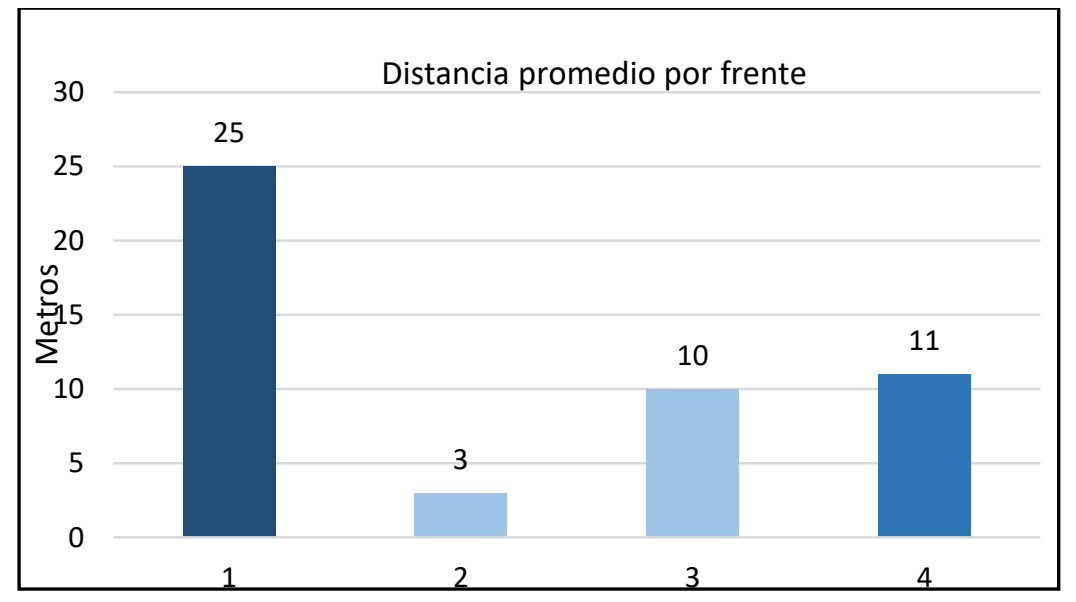

Figura V: Distancia entre la fuente de abastecimiento y los establecimientos observados. Fuente: Elaboración propia

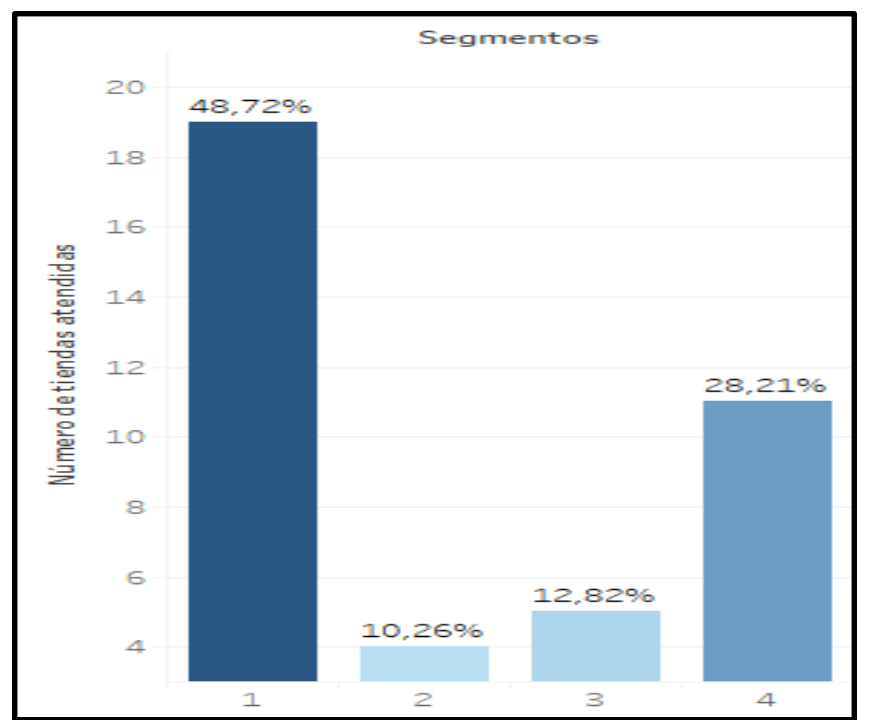

Figura VI: Proporción de entregas según frente. Fuente: Elaboración propia 


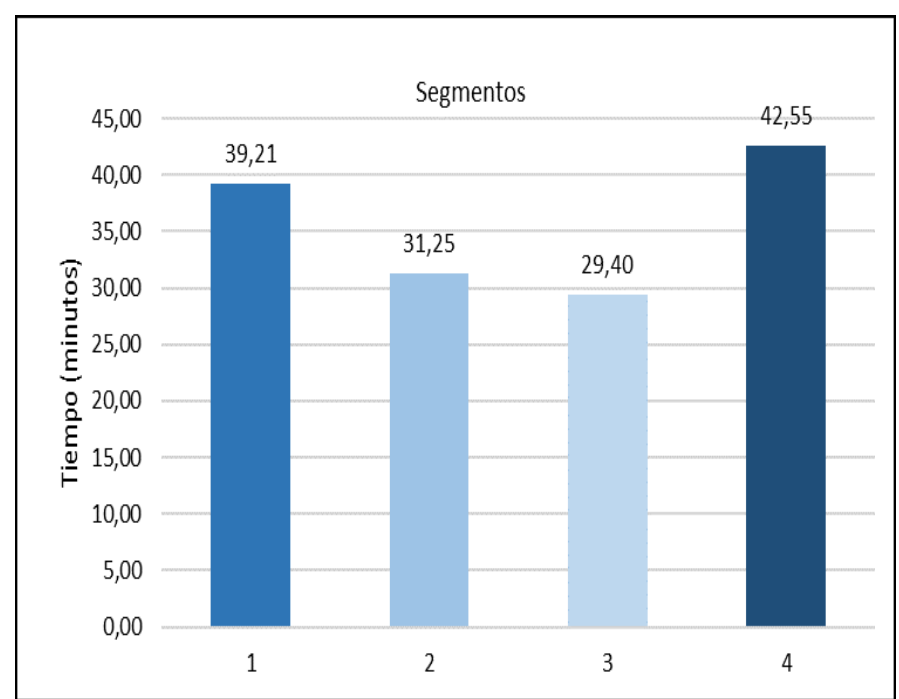

Figura VII: Tiempo en minutos para realizar las entregas. Fuente: Elaboración propia

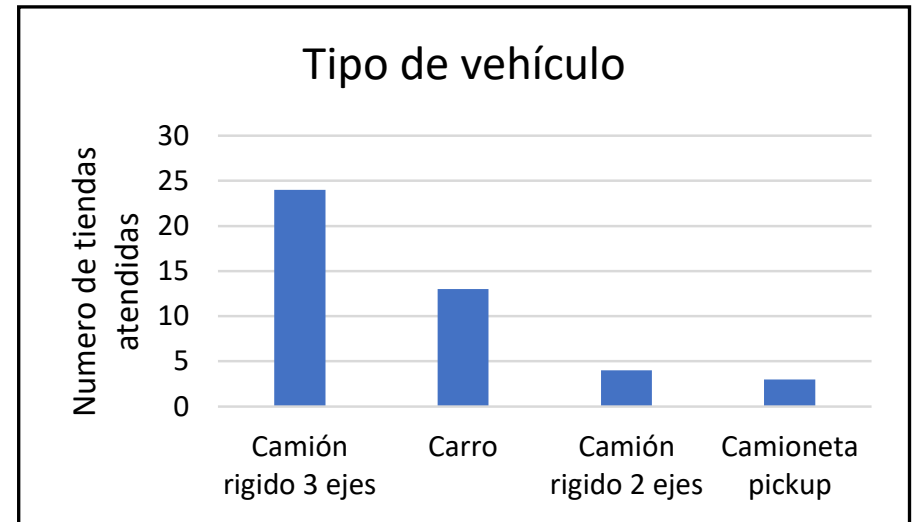

Figura VIII: Tipo de vehículo usado en las entregas. Fuente: Elaboración propia

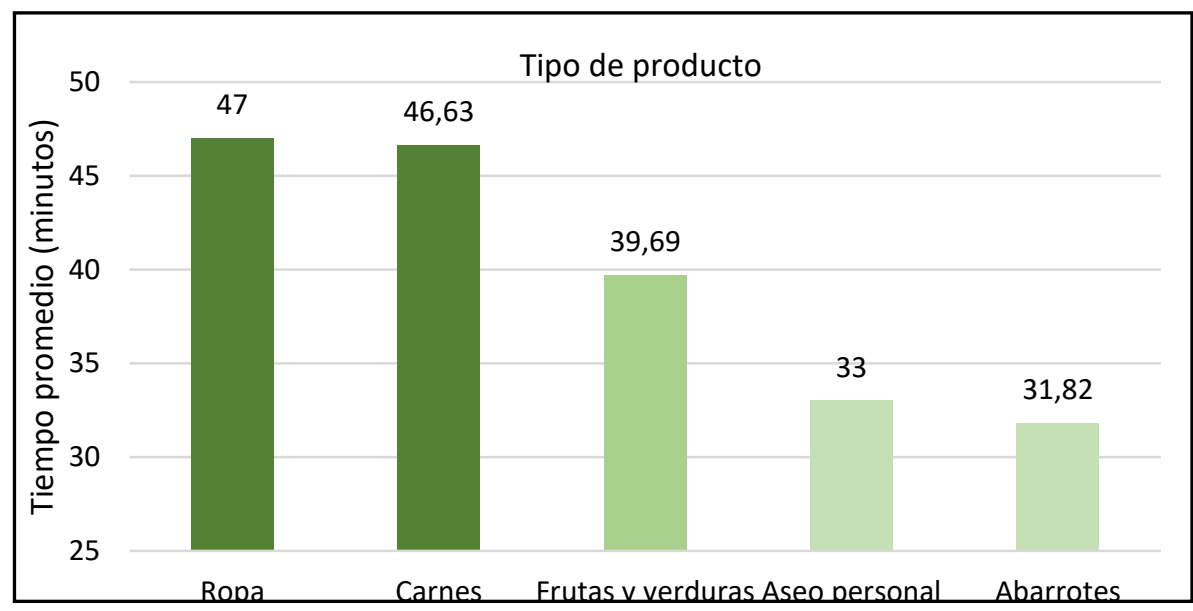

Figura IX: Tiempo promedio de duración de entrega por tipo de producto. Fuente: Elaboración propia

Memoria Investigaciones en Ingeniería, 2019. №17(1). pp 31-46

https://doi.org/10.36561/ING.17.3

ISSN 2301-1092 • ISSN (en línea) 2301-1106 
5.5. Disrupciones. - Durante el periodo de observación, se apreciaron setenta y nueve disrupciones. Las disrupciones más frecuentes fueron los vehículos estacionados en doble fila, estacionamiento en ambas vías, interrupciones del tráfico y de peatones. Las interrupciones vehiculares en cada uno de los frentes fueron de 64\%, 57\%, 62,5\% y $75 \%$. La Figura $X$ muestra las interrupciones por frente y tipo.

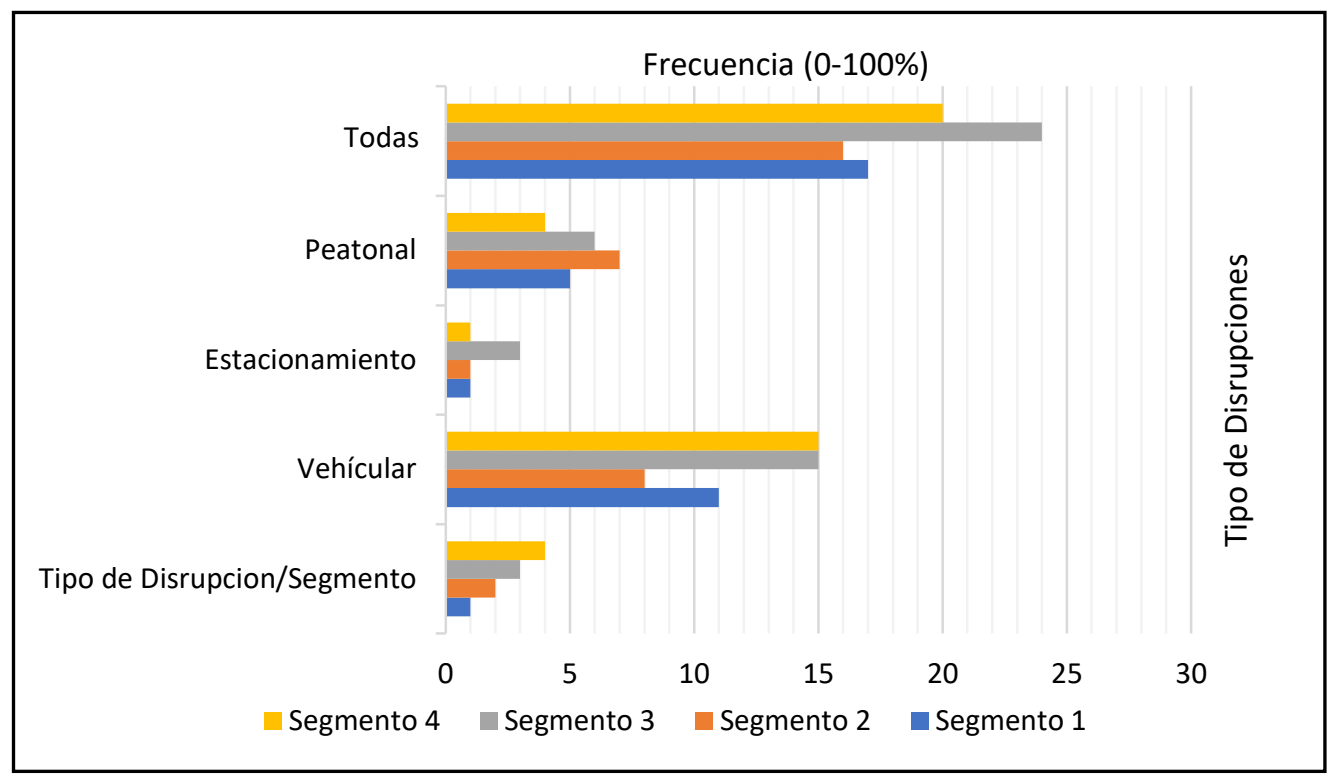

Figura X: Tipo de disrupciones por frente. Fuente: Elaboración propia

Flujo de vehículos y personas. - El flujo vehicular se observó entre las 08:00 y las 17:00 horas. En la Figura XI se muestra un flujo en los frentes 1 y 2; con 101 vehículos/hora y 55 personas/hora. Los frentes 3 y 4 tuvieron 138 vehículos/hora y 71 personas/hora.

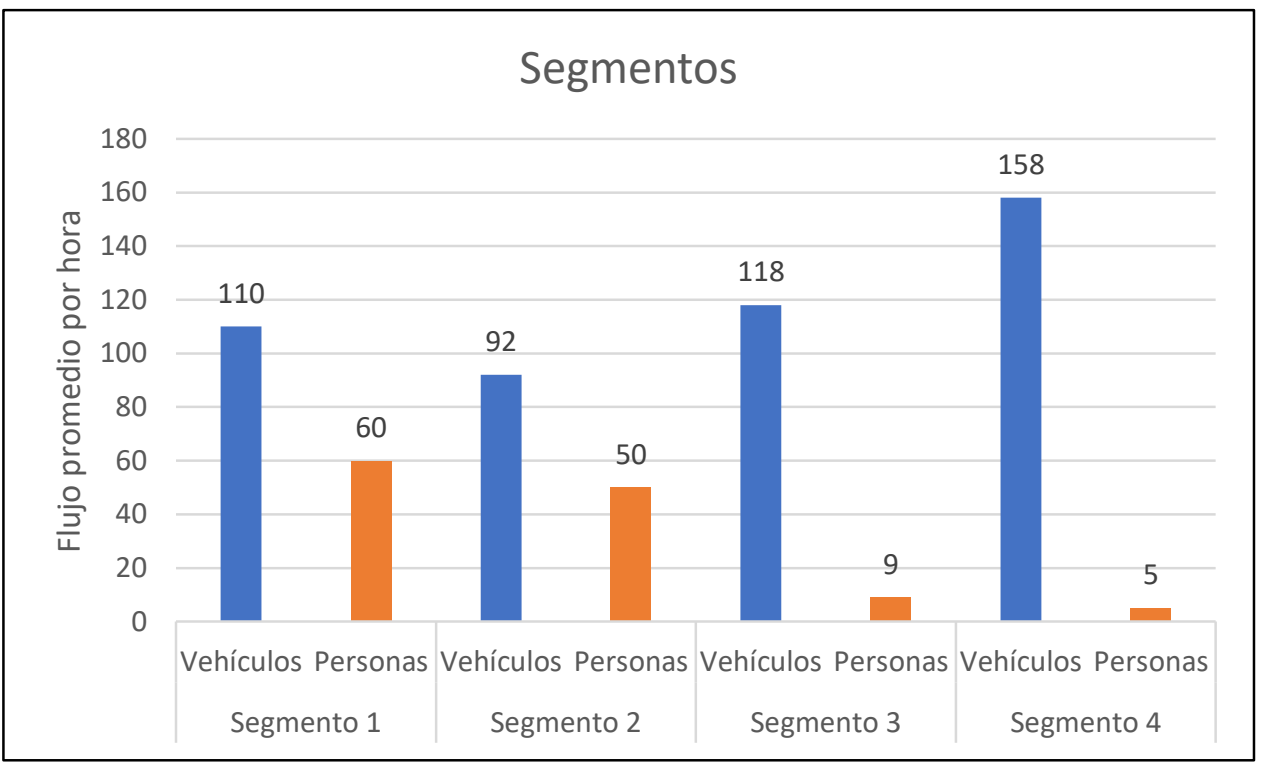

Figura XI: Flujo de tráfico promedio entre frentes. Fuente: Elaboración propia

Memoria Investigaciones en Ingeniería, 2019. №17(1). pp 31-46

https://doi.org/10.36561/ING.17.3

ISSN 2301-1092 • ISSN (en línea) 2301-1106 
6. Acerca de las bahías de carga y descarga. - El área comercial de Chosica no posee bahías de carga y descarga de mercancías. Los locales comerciales no poseen un estacionamiento privado; y el uso del espacio público está en constante competencia. La zona tiene 35 estacionamientos y, en muchos casos, ocupados por los comerciantes desde el inicio de la jornada laboral. Las Figuras XII y XIII muestran algunas de las disrupciones por la ausencia de las bahías de carga y descarga.

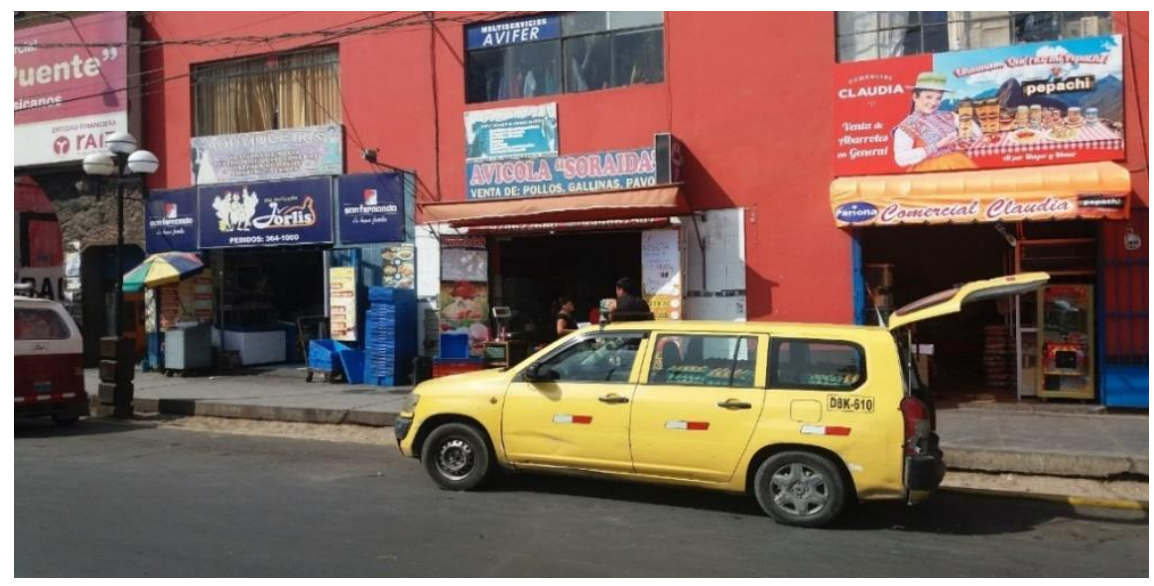

Figura XII: Auto estacionado en la vía para abastecer su mercancía a tienda minorista. Fuente: Elaboración propia

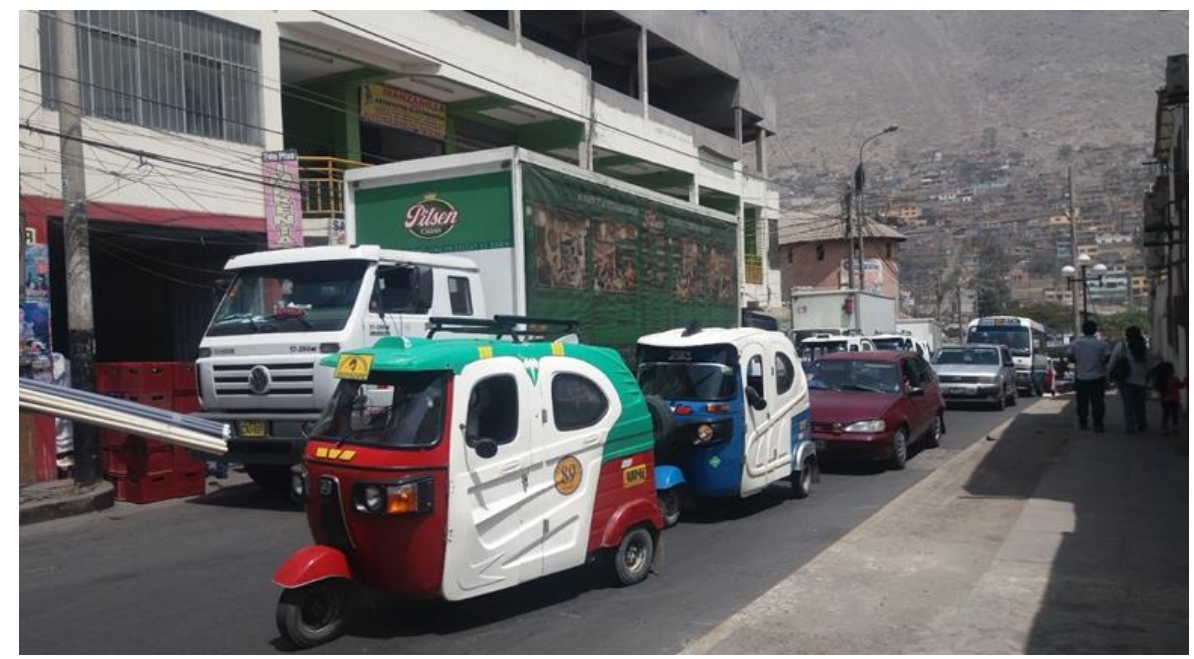

Figura XIII: Camión estacionado en la vía para abastecer su mercancía a tienda minorista. Fuente: Elaboración propia

7. Políticas propuestas para mejorar la distribución de mercancías en la zona. - La falta de infraestructura, el desorden en la distribución de mercancías y la congestión vehicular en la zona de Chosica evidencian una inexistente planificación urbana. En esta sección se presentará una serie de estrategias para la gestión de mercancías y entregas con el objetivo de usar, de manera adecuada, el espacio púbico en base a la caracterización de la zona de estudio y el impacto en los ciudadanos.

7.1. Implementación de bahías de carga y descarga. - La política de asignación del espacio en la vía para actividades de carga y descarga sería recomendable en el tercer $\mathrm{y}$ cuarto frente, por el estacionamiento en doble fila para estas actividades $\mathrm{y}$, muchas 
veces, ambas vías, causado por la falta de espacios dedicados. Una eficaz implementación de esta política reduciría en un 63,64\% la cantidad de disrupciones totales, apoyando a un mejor flujo vehicular, el cual mejoraría en un promedio de 7 minutos por viajes en la zona. El objetivo es reducir el tiempo de las disrupciones y evitar sanciones a los transportistas. Actualmente, se requiere una distancia mínima de 3,0 a 4,2 metros para abastecer locales [35], el segundo frente cumple con esta norma. Esta política se ha aplicado en San Francisco, donde tomó la decisión de ampliar las aceras y designar el uso compartido de la vía para actividades de estacionamiento y carga. En Washington DC, un estudio de flete en la vía recomendó proporcionar espacios de estacionamiento y carga más largos [36]. Otras recomendaciones son aumentar el tamaño de las áreas de carga a 30 metros lineales [20]. El Departamento de Transporte de la ciudad de Nueva York aumentó los estacionamientos de carga asignados a vehículos comerciales e instaló parquímetros. La industria del transporte de mercancías ha reaccionado favorablemente a esta política y ha facilitado el flujo de los productos [20]. En Lima, se está empezado a implementar estas bahías [11] con un efecto en la competitividad económica de las ciudades al reducir los costos de transporte de carga en estos sistemas urbanos [37].

7.2. Ampliación de vías. - Los nuevos o mejorados caminos consideran los radios de giro amplio de los camiones [38]; los camiones grandes no pueden girar a la derecha sin interferir con el tráfico de sentido contrario o cortan las aceras. Un ejemplo de esta iniciativa son los corredores de carga, implementados en el Georgia Logistics en Atlanta [39]. Las vías en la zona de estudio son de un solo sentido. La ampliación de las vías permitiría reducir el tráfico, además los vehículos de carga tendrían más espacio para transitar, realizar los repartos y abordar con más seguridad los giros amplios. La ampliación de las vías reduciría aproximadamente en un 26,50\% la cantidad de disrupciones totales relacionadas al uso vehicular [39]. Sin embargo, las implementaciones de proyectos viales en el Perú son altamente ineficientes con un promedio de $36,78 \%$ de atraso acumulado [40].

7.3. Implementación de rampas. - La construcción de rampas en las aceras para transportar mejoraría la eficiencia de las actividades de carga y descarga [38]. Estas rampas facilitan a los conductores la entrega de grandes cantidades de carga y reduce el tiempo en las áreas de estacionamiento y carga. Las rampas también permiten descargar los productos para una ubicación general, dividir la carga y distribuirla a varios lugares cercanos, además de contar con un único lugar de carga y descarga [20]. Del total de entregas, el 46,15\% se realizó con carretillas. Estas se concentraron en el primer y cuarto frente. La implementación de rampas permitiría mejorar la productividad del abastecimiento a un bajo costo.

7.4. Regulaciones para las actividades de carga y descarga. - La implementación de bahías de carga y descarga con zonas de usos flexibles [20] fue implementada en el programa del Department of Transportation (DOT) de la ciudad de Nueva York [41]. Estas regulaciones podrían mejorar el sistema de planificación de las empresas; y reduciría el tiempo de descarga dependiendo de la ventana de abastecimiento y su posterior costo. La viabilidad de la política va a depender de las regulaciones a los operadores logísticos. Esta opción ha demostrado, a nivel mundial, una mejora en la gestión en un $69 \%$ [37]. Sin embargo, si bien la restricción podría reducir la congestión de transporte de carga y mejorar la seguridad y movilidad de la zona, su efectividad

Memoria Investigaciones en Ingeniería, 2019. №17(1). pp 31-46

https://doi.org/10.36561/ING.17.3

ISSN 2301-1092 • ISSN (en línea) 2301-1106 
podría verse limitada por la falta de control y de incentivos. Esta fue una observación en el perfil logístico de Quito [5].

7.5. Sistemas de reserva de plazas de estacionamiento. - Los sistemas inteligentes de transporte permiten reservar plazas de estacionamientos a los conductores, y ayudan a la asignación y el uso de espacios de estacionamiento [20]. Esta política, bien implementada, puede optimizar el transporte de carga, como ya se evidencio en la ciudad de Toyota [26] Esta política sería factible en zonas altamente transcurridas, tales como el primer y cuarto frente, por las entregas. En el primer frente, un 65\% de entregas se dan antes del mediodía, en comparación con $80 \%$ del cuarto frente. Esto genera una temprana congestión de vehículos de carga, generando un mayor tráfico y cantidad de disrupciones. Se estima que, si el sistema de reservas fuera eficaz, la cantidad de entregas se regularía en cuanto horas de llegada (un 50\% en la mañana y un $50 \%$ en la tarde), tal y como ocurre en los frentes 2 y 3 , mejorando en $12,82 \%$ de las distribuciones totales.

7.6. Carriles de uso exclusivo para camiones. - Las vías en la zona comercial de Chosica se podrían convertir en una vía exclusiva para la circulación de carga. Los carriles exclusivos permiten mejorar las operaciones, con una mayor confiabilidad de los tiempos de entrega y menores impactos ambientales y riesgo de accidentes. El desarrollo de carriles exclusivos podría darse con una adecuada planificación de las instalaciones urbanas [42]. Las experiencias y lecciones aprendidas de la implementación previa, problemas típicos que enfrentan los planificadores al principio del proceso de planificación, así como y un marco y métodos para evaluar los beneficios e impactos de las instalaciones dedicadas para camiones [20] hacen posible lograr una circulación efectiva y eficaz.

8. Conclusiones. - Las ciudades son ambientes creados por el hombre, con una interacción de múltiples sistemas con el fin de satisfacer las necesidades de los ciudadanos, dentro de una competencia constante por el uso de los espacios públicos de manera eficiente. Uno de los sistemas relacionados con el transporte y distribución es la gestión de carga y entrega de mercancías; en un sistema donde el mercado debe recibir las mercaderías, y los transportistas deben usar los espacios públicos considerando infracciones de tránsito, uso de las vías públicas y generación de gases contaminantes.

Este artículo presentó el caso de Chosica, una ciudad en Lima; y propuso alternativas para las entregas de mercancías con un uso multimodal del espacio público que contiene la implementación de bahías de carga y descarga; ampliación de las vías; implementación de rampas; regulación a las actividades de carga y estacionamiento; sistema de reserva de plazas de estacionamiento; y regulación de carriles de carga. La planificación urbana, incluyendo a todos los actores, permitió tener una ciudades más ordenada y eficiente en la distribución de mercancías. Se puede concluir que Las bahías de carga y descarga cumplen un rol importante para agilizar el flujo de las operaciones, permitirá mejorar el flujo de entrega de las mercancías a un bajo costo y de sencilla implementación.

Las tres políticas siguientes permiten, en conjunto, reducir en gran magnitud los costos de transporte de carga de la ciudad: (1) las bahías de carga y descarga reducirían en un 63,64\% las interrupciones en el flujo vehicular; y reduciría el tiempo de los viajes en la zona a siete minutos, en promedio; (2) La implementación de rampas y el sistema de reservas reduciría el tiempo de respuesta en un 12,82\%; y (3) las regulaciones de la carga y descarga permitirían planificar las actividades de las zonas. Sin embargo, la

Memoria Investigaciones en Ingeniería, 2019. №17(1). pp 31-46

https://doi.org/10.36561/ING.17.3

ISSN 2301-1092 • ISSN (en línea) 2301-1106 
demanda por vías públicas seguirá siendo grande y esto representa un límite a la reducción del total de ineficiencias.

Para lograr un impacto en la zona comercial de Chosica, las políticas públicas deben contar con la participación de los stakeholders, sector público con los gobiernos municipales y el Ministerio de Transportes y Comunicaciones; el sector público con los transportistas, comerciantes, peatones, empresas de servicios y el sector académico, con una solución estratégica basada en el consenso con la participación, la colaboración y la asociación de las entidades.

\section{Referencias}

[1] Grupo Banco Mundial, Perú Hacia un Sistema Integrado de Ciudades. Lima: Banco Mundial, 2016.

[2] P. Hall, M. Hesse, Cities, Regions and Flows. London: Routledge, 2012.

[3] E. Blanco, A. Bateman, y D. Merchán, "Urban Metrics for Urban Logistics: Building an Atlas for Urban Freight Policy Makers," en International Conference on Computers in Urban Planning and Urban Management, 2015.

[4] E. Blanco, y D. Merchán, "Desafíos para la movilidad de carga en zonas de alta congestión," Economía Industrial, no. 400, pp. 13-19, 2016.

[5] D. Merchán, "El perfil logístico de Quito," Cuestiones Urbanas, vol. 5, no. 1, pp. 93-135, 2015.

[6] L. Dablanc, "Goods transport in large European cities: difficult to organize, difficult to modernize," Transportation Research Part A: Policy and Practice, vol. 41, no. 3, pp. 280 285, 2007 doi:10.1016/j.tra.2006.05.005

[7] L. De Oliveira, "Diagnóstico das vagas de carga e descarga para a distribuição urbana" Journal of Transport Literature, vol. 8, no.1, pp. 178-209, 2014.

[8] L. Dablanc, Freight transport for development toolkit: urban freight. Washington: The International Bank for Reconstruction and Development / The World Bank, 2010.

[9] S. Greenbaum, Loading dock. NIBS: Washington, 2016.

[10] L. Dablanc, Freight transport for development toolkit: Urban freight. Washington: The World Bank, 2009.

[11] Massachusetts Institute of Technology, km2 Guide, 2018.

[12] M. Jaller, J. Holguín-Veras, y S. Hodge, "Parking in the city: Challenges for freight traffic," Transportation Research Record Journal of the Transportation Research Board, vol. 2379, no.1, pp. 46-56, 2013.

[13] J. Holguín-Veras et al., "An investigation on the effectiveness of joint reciver-carrier policies to increase truck traffic in the off-peak hours," Networks and Spatial Economics, vol. 8, no. 4, pp. 327-54, 2008. doi:10.1007/s11067-006-9011-6.

[14] Ministerio de Comercio y Turismo del Perú, Análisis integral de la logística en el Perú. Lima: Ministerio de Comercio y Turismo del Perú, 2016.

[15] Ministerio de Economía y Finanzas del Perú, Mejora de la gestión urbana y territorial. Lima: Ministerio de Economía y Finanzas del Perú, 2017.

[16] I. Thomson y A. Bull, La congestión del tránsito urbano: causas y consecuencias económicas y sociales. Santiago de Chile: CEPAL, 2001.

[17] Municipalidad de Miraflores, Plano urbano distrital. Lima: Municipalidad de Miraflores, 2015.

[18] J. Gonzalez-Feliu et al., Sustainable Urban Logistics: Concepts, Methods and Information Systems. Berlin: Springer Berlin Heidelberg, 2014. doi:10.1007/978-3-642-31788-0

[19] Institut Cerdà, Logística urbana. Ciudad y mercancias. Barcelona: Marge Books, 2010.

Memoria Investigaciones en Ingeniería, 2019. №17(1). pp 31-46

https://doi.org/10.36561/ING.17.3

ISSN 2301-1092 • ISSN (en línea) 2301-1106 
[20] J. Holguín-Veras et. al., "Improving Freight System Performance in Metropolitan Areas: A Planning Guide,” Transportation Research Board, Washington, NCFRP Rep. 33, 2015.

[21] Un-Habitat, Planning and Design for Sustainable Urban Mobility. Abingdon: Routledge, 2013.

[22] E. Lindholm y M. Blinge, "Assessing knowledge and awareness of the sustainable urban freight transport among Swedish local authority policy planners," Transport Policy, vol. 32, pp. 124-131, 2014.

[23] M. Nourinejad et. al. Truck parking in urban areas: application of choice modelling within traffic simulation. Woodstock: Canadian Transport Research Forum, 2013.

[24] J. Holguín-Veras, I. Sánchez-Díaz, y M. Browne, "Sustainable urban freight systems and freight demand management", Transportation Research Procedia, vol. 12, pp. 40-52, 2016.

[25] J. Holguín-Veras et al, "Direct impacts of off-hour deliveries on urban freight emissions," Transportation Research Part D: Transport and Environment, vol. 61, pp. 84-103, 2018.

[26] PIARC, Public Sector Governance of Urban Freight Transport. Paris: PIARC: 2012.

[27] E. Ballantyne y M. Lindholm, "Identifying the need for freight to be included in local authority transport planning," en Sustainable urban logistics: Concepts, methods and information systems, Jesus Gonzalez-Feliu, Frédéric Semet, Jean-Louis Routhier, Eds. Berlin: Springer Berlin Heidelberg, 2014, pp. 37-48.

[28] T. Crainic, N. Ricciardi, y G. Storchi, "Models for evaluating and planning city logistics systems," Transportation science, vol. 43, no. 4, pp. 432-454, 2009.

[29] A. May et al., "An option generation tool for potential urban transport policy packages," Transport Policy, vol. 20(C), pp. 162-173, 2012.

[30] R. Danielis, L. Rotaris, y E. Marcucci, "Urban freight policies and distribution channels: a discussion based on evidence from Italian cities," European Transport, vol. 46, no. 46, pp.114-146, 2010.

[31] Massachusetts Institute of Technology, Megacity Logistic Lab: Last Mile. Cambridge: Center of Transportation and Logistics, 2013.

[32] OBNASEC, Ficha informativa sobre seguridad ciudadana del distrito de LuriganchoChosica. Lima: OBNASEC, 2016.

[33] Instituto Nacional de Estadística e Informática. Perú: Población. Lima: Instituto Nacional de Estadística e Informática, 2017.

[34] Open Street Maps, 2018.

[35] Instituto Nacional de Seguridad e Higiene en el Trabajo. Muelles de Carga y Descarga: Seguridad. NTP 1.076, 2013.

[36] E. Jones, A. Chatterjee, y R. Marsili, “A Collaborative Plan for Curbside Freight Delivery in Washington D.C.," ITE Journal, vol. 79, no. 5, pp. 22-25, 2009.

[37] I. Sánchez-Díaz et al., Urban Freight Initiatives: State of the Art and State of the Practice. Gothenburg: Urban Freight Platform, 2017.

[38] K. Ogden, Urban Goods Movement: A Guide to Policy and Planning. Farnham: Ashgate Publishing Company, 1992.

[39] Departamento de Transporte de Georgia. Statewide Truck Lane Needs Identification Study, 2007, Departamento de Transporte de Georgia

[40] Contraloría General de la República. Informe de Seguimiento y Monitoreo de Proyectos N ${ }^{\circ}$ 00010-2017-CG/MPROY. Lima: Contraloría General del Perú, 2017.

[41] Departamento de Transporte de Nueva York. Citywide Congested Corridors Study. Nueva York: Departamento de Transporte de Nueva York, 2012.

[42] J. Douglas, Handbook for Planning Truck Facilities on Urban Highways. New York: Parsons Brinckerhoff, 2004. 